Bulletin UASVM Food Science and Technology 70(1)/2013, 8-15

ISSN-L 2344-2344; Print ISSN 2344-2344; Electronic ISSN 2344-5300

\title{
Fingerprinting Food Supplements and Their Botanical Ingredients by Coupled UV/Vis/FTIR Spectrometry
}

\section{Anca BACIU ${ }^{2)}$, Floricuța RANGA ${ }^{1)}$, Florinela FETEA $^{1)}$, Simona ZAVOI ${ }^{1)}$, Carmen SOCACIU ${ }^{1,2^{*}}$}

${ }^{1)}$ Department of Biochemistry, University of Agricultural Sciences and Veterinary Medicine, Faculty of Food Science and Technology, Cluj-Napoca, Romania

${ }^{2)}$ CCD-BIODIATECH, Proplanta SRL Cluj-Napoca, Romania

carmen.socaciu@usamvcluj.ro

\begin{abstract}
Medicinal plants are used as ingredients for a large variety of herbal supplements. Their quality and safety versus efficacy, according to present legal requirements, need to meet the minimum quality criteria to support their use. Specific biomarkers to evaluate and screen their authenticity are phenolic derivatives, phtosterols, lipids or alkaloids. We report here the data obtained for two herbal food supplements (A and B) obtained from different mixtures of plants: Taraxacum officinalis, Cynara scolimus Silybum marianum as ingredients for product A) and Hypericum perforatum, Chelidonium majus and Lycopodium clavatum as ingredients for product B).

The combination of UV-Vis and FTIR spectrometry allowed a specific fingerprint of biomarkers in individual plants and derived supplements (A and B), by discriminating the specific areas and peaks of individual plants and mixtures, the significant differences between the methanolic and water extracts.

The data were compared using chemometry (PCA and Cluster analysis).

Using Vis spectrometry combined with FTIR peak intensities at $1732 \mathrm{~cm}^{-1}$ and calibration with gallic acid, the total phenolics concentrations ranged from 5.31 to $9.58 \mathrm{mg}$ gallic acid eq/ml methanol, with a positive and significant correlation between the two methods $\left(R^{2}=0.979\right)$. The phenolics' concentrations were 2.5 to 4 times lower in water extracts comparing with methanol extracts of products A and B. Finally, we assume that herbal supplements can be adequately characterized for their quality and safety by combined UV-Vis spectrometry/FTIR spectrometry, with good, fast and cheap informations about the main biomarkers of authenticity.
\end{abstract}

Keywords: herbal supplements, fingerprint, UV-Vis, FTIR, chemometry, authenticity

\section{INTRODUCTION}

Medicinal plants are used since centuries, as such or as ingredients for a large variety of herbal mixtures as food supplements. Their quality and safety versus efficacy, according to present legal requirements, need to meet the minimum quality criteria to support their use worldwide (Ramawat et al., 2009; Verpoorte, 2009; Gong et al., 2009). Meanwhile, the natural heterogeneity of the plants from herbal medicines determine fluctuations of the type and concentration of different phytochemicals found in medicinal plants, dependent on ontogenic or genotypic factors, influenced by environment, age, time of harvesting, drying and storage (Wichtl, 2004). These fluctuations determine also difficulties in identifying and quantifying the active molecules responsible for the therapeutic effect.

The main phytochemicals, generally used as markers of plant taxonomy and authenticity are secondary metabolites such as carotenoids/chlorophylls/phenolic acids/flavonoids/anthocyanins or monoterpenoids/unsaturated fatty acids/ phytosterols, but also, in some specific cases, saponins, lignans and indoles, thiols and glycosinolates, vitamins 
A, C, E. All these classes of molecules have specific roles in plants either for attraction/defence mechanisms or for their antioxidant/antibiotic action. Their functionality and beneficial action for human health, proved to be largely dependent on their supramolecular architecture and environment, solubility and redox potential, stability, and bioavailability (Socaciu et al., 2009). The appropriate identification of the herbs' quality and authenticity is successfully determined either by chromatographic (HPLC or GC) or by simpler methods such as Infrared spectroscopy (FTIR) combined with biostatistical tools (cluster analysis and linear correlation coefficient analysis). The last years, the integrative concept of plant metabolomics involve the use of advanced methodologies for identification and quantification of individual molecules (by MS or NMR) and facilitates an improved understanding of dynamic biochemical composition within the living systems, monitoring plant quality characteristics, identifying potential biochemical markers in individual plants and in mixtures, detecting product authenticity and possible adulterations. A four steps procedure to evaluate plant or food metabolic profile using complementary methods was reported (Socaciu et al., 2009).

These fingerprinting approaches are often combined with multivariate analyses to get most out of the data and need validation. According to plant metabolomics methodology, the hyphenated complementary analytical techniques of liquid or gas chromatography coupled with MS or NMR detectors (GC/MS, LC/MS, LC/MS/MS, LC/NMR, LC/PDA/MS/FTIR in specific platform are yet functional in specialized EU centers, providing spectral libraries and metabolomic databases (www.phenol-explorer.eu; www.liberherbarum.com; www.metabolomics-lab.com; www.kegg.jp )

Beside these sophisticated methods applied to identify or discover new biomarkers of plant recognition,(Socaciu et al., 2009; Mattoli, 2011; Baranska and Schultz, 2006; Fan et al., 2006; Gong et al., 2006; Duron et al., 2009; Giri et al., 2010) many laboratories use rapid and cheaper, available methods to fingerprint and authentify the composition of such products, e.g. Fourier transform infrared spectroscopy (FTIR), a convenient method to fingerprint herbal medicines, especially when is validated with chromatographic (HPLC-UV or -PDA) analysis (Baranska and Schultz, 2006; Duron et al., 2009).

Among the most important phytochemicals found in medicinal plants are the phenolic derivatives, a large family of secondary metabolites with various roles in plant defense, with antioxidant activity and beneficial health effects (Scalbert et al., 2005). Until now, there were elaborated many analytical protocols to separate, identify and quantify phenolic acids, flavonoids and their glucosides, catechins, tannins (Santos-Buelga and Williamson, 2003; Singleton, 1999). The comparative FTIR/HPLC-PDA profile of European plants (Echinaceea sp. Melissa off.) combined with chemometric analysis, or other plants from Romanian flora in relation with their phenolic content and antioxidant capacity was recently reported (Socaciu et al., 2009; Spiridon et al., 2011).

In this study we investigated two different herbal medicines with hepatoprotective potential, based on the traditional, longtime use of their ingredients (medicinal plants). Foe example, dandelion (Taraxacum officinalis) is an old remedy in the therapy of liver diseases due to ,taraxacin” a mixture of eudesmanolide and germacranolide derivatives and phenolics (apigenol and luteolin) (Schutz et al., 2005). Artichocke (Cynara scolimus) is known as colagogue and liver regenerative due to cynarin (Wang et al., 2003). Milk thistle (Silybum marianum) acts as hepatocyte activator by ,silymarin” flavolignan complex and taxifolin, a flavonid (Ding et al., 2001; Minakhmetov et al., 2001). St John's wort (Hypericum perforatum) is known to contain hypericin, hyperforin and phenolics, mainly flavonoids (rutoside) (Mulinacci et al., 1999; Barnes et al., 2001). Celandine (Chelidonium majus ) is rich in chelidonin with colecystokinetic and antispastic effects, antihepatotoxic and 
anticancerigenic. Wolf's claw (Lycopodium clavatum) contains alkaloids and lignans, triterpens and flavonoids with good effects on liver tumor inhibition, anti-inflammatory and antimicrobial action (Pedersen and Ollgaard, 1982).

We report here experimental data obtained by comparative fingerprints of methanol and aqueous extracts of two herbal medicines (A and B) comparative to their individual plant ingredients. The first herbal product (A) was made from a mixture of dandelion, artichoke and milk thistle and the second one (B) contained St John wort, celandine and Wolf's claw.

We used in parallel UV-Vis spectrometry to fingerprint extracts' composition and to quantify total phenolics and Fourier Transform Infrared spectrometry for middle region (FTMIR $=650-4000 \mathrm{~cm}^{-1}$ ). To validate the quantitative evaluation of phenolics by FTIR as a reliable quantification method for phenolics, calibrations with gallic acid were made and compared with Vis spectrometric data.

\section{MATERIALS AND METHODS}

\section{Plant materials and sample preparation}

Six medicinal plants were provided from a wild environment from Transylvani, Romania. These were numbered as follows: 1 - Taraxacum officinalis (Dandelion), 2- Cynara scolimus (artichoke), 3-Silybum marianum (milk thistle), 4- Hypericum perforatum ( $\mathrm{St}$ John's wort), 5- Chelidonium majus ( Celandine), 6- Lycopodium clavatum (Wolf's claw). Excepting milk thistle seeds, the other plants were used as a whole plant, without roots.

The dried plant samples were grounded, powdered and submitted to extraction, using duplicated samples from each plant. Two types of herbal supplements were obtained: A _ by mixing powders of dandelion, artichoke and milk thistle, 1:1:1 (weight ratios), and $\mathrm{B}$ - from St John's wort, Celandine and Wolf's claw, 1:1:1 (weight ratios).

Aliquots of $15 \mathrm{~g}$ individual plant powder or herbal mix (A or B) were mixed with 85 $\mathrm{ml}$ solvent (methanol $90 \%$ in water, containing $1 \% \mathrm{HCl}$ ) and sonicated for $30 \mathrm{~min}$ in a bath and centrifuged $10 \mathrm{~min}$ at $2000 \mathrm{rot} / \mathrm{min}$. The supernatant was collected and filtered through a $0.45 \mu \mathrm{m}$ filter, representing the methanolic extract (ME). In parallel, for A and B products we made a water extract (WE), using the same concentration of $15 \%$ plant by the same extraction steps.

\section{UV-Vis spectral analysis}

The UV-Vis spectra of all extracts were recorded (700-200 nm) using a Jasco V 530 spectrophotometer. From each spectra, the specific fingerprint was recorded, as well the maxima wavelengths, specific to phenolic derivatives $(280$ and $330 \mathrm{~nm})$. In these conditions, we compared the fingerprint of $\mathrm{ME}$ from individual plant extracts with the fingerprint of supplements A and B in both ME and WE.

\section{Determination of Total phenolics by Vis spectrometry}

Aliquots of $1 \mathrm{ml} \mathrm{ME}$ extracts from each plant and A /B supplements were mixed with water, $5 \mathrm{ml}$ Folin reagent, homogenized with $15 \mathrm{ml}$ Natrium carbonate 7,5\%, according to the method of Singleton (1999). After $2 \mathrm{hrs}$, the absorbance at $\lambda=750 \mathrm{~nm}$ was recorded against a blank. In parallel, a calibration curve was built with pure gallic acid as a standard, in the range of 0.0625 to $1 \mathrm{mg} / \mathrm{ml}$ methanol. Considering the curve equation $\mathrm{y}=0.515 \mathrm{x}+0.0504$, with $\mathrm{R}^{2}=0.9993$, the total phenolic concentrations of all samples were calculated. 


\section{FTIR analysis}

The FTMIR spectra of fingerprint regions, specific for ME extracts of the individual plants 1-6 and herbal supplements A and B were determined using a FTIR spectrometer (Shimadzu Prestige 2, Apodization: Happ-Genzel spectrometer). Each spectrum was recorded in the MIR region (4000 to $650 \mathrm{~cm}^{-1}$ ) with 64 scans were accumulated for each spectrum using the Horizontal Attenuated Total Reflection (HATR) device. The spectra were processed by IR solution Software Overview (Shimadzu) and OriginR 7SR1 Software (OriginLab Corporation, Northampton, USA).

To evaluate quantitatively the phenolic concentration of each sample, the FTIR spectra were calibrated with gallic acid (0.0625 to $1 \mathrm{mg} / \mathrm{ml}$ methanol). Two different calibration curves were made previously, considering either the total peak areas of fingerprint region (1610-1743 $\mathrm{cm}^{-1}$ ), or the absorption intensity at $1610 \mathrm{~cm}^{-1}$ or at $1732 \mathrm{~cm}^{-1}$. The best correlation IR absorption intensity $=\mathrm{f}$ ( gallic acid) was found considering the intensity at $1732 \mathrm{~cm}^{-1}$, with a curve equation $\mathrm{y}=0.0819 \mathrm{x}+0.0184$ and $\mathrm{R}^{2}=0.9994$ (data not shown).

\section{RESULTS AND DISCUSSIONS}

\section{UV-Vis spectroscopy data}

Fig. 1 shows the comparative UV spectral fingerprints $(200-500 \mathrm{~nm})$ of the herbal supplements A and B in ME.

In these conditions, each ingredient of the product A showed its specific spectral shape. Previously we made the spectra of all ingredients (Zavoi et al., 2011a), the dandelion extract showed three main peaks, at 280, 330 and $390 \mathrm{~nm}$, while artichocke had also three absorption regions, more intense in the range 240-280 nm. Specifically, milk thistle seed extract had a higher peak absorption at $280 \mathrm{~nm}$ with a shoulder located at $320 \mathrm{~nm}$ and no absorption at $390 \mathrm{~nm}$. In the spectra of product A one can identify the shape of a dominant peak originating from milk thistle $(270-290 \mathrm{~nm})$, a large peak from 300 to $400 \mathrm{~nm}$ and a peak at $420 \mathrm{~nm}$ attributed to oxidized forms of phenolics (chinones).

The plants used as ingredients for product B have had different fingerprints: St John wort had a dominant peak in the region $270-290 \mathrm{~nm}$ and at $520 \mathrm{~nm}$, as indicator of Hypericin. Celandine had three absorption regions, at 270-290 nm, 330-350 nm and $390 \mathrm{~nm}$, while wolf's claw extract showed no absorptions at $280 \mathrm{~nm}$, but large peaks between 320 and 350 $\mathrm{nm}$ and $400-420 \mathrm{~nm}$. Looking to the spectra shape of product B extract, one can identify a combination of peaks in the region 270-290 nm ( from St John wort and celandine) combined with the absorption regions specific to wolf's claw, at 320-350 nm and 400-420 nm (Zavoi et al., 2011).
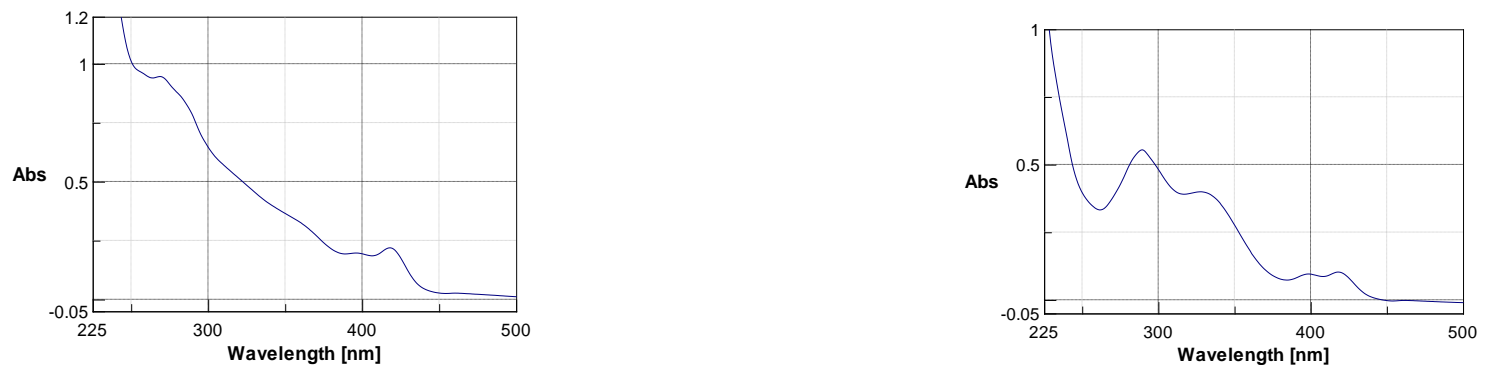

Fig. 1. Comparative UV-VIS spectral fingerprints $(200-500 \mathrm{~nm})$ for methanol extracts (ME) products A (left) and B (right)

When the ME extract was compared with WE, lower extraction yields were obtained in water, e.g. 2.5 times lower for A and 4 times lower for product B, suggesting that B has a 
less polar composition than A. We can assume that UV spectra fingerprints give general information about the polar phenolics' solubility and ratios between their subclasses.

According to Tab. 1, dandelion, artichoke and celandine extracts, followed by St John wart extract, were richer in phenolics (from 9.30 to $8.38 \mathrm{mg}$ gallic acid eq. $/ \mathrm{ml}$ ME) while wolfs' claw and milk thistle were less $(7.69$ and $6.66 \mathrm{mg}$ gallic acid eq. $/ \mathrm{ml} \mathrm{ME}$, respectively. The product $\mathrm{A}$ had higher concentration $(9.37 \mathrm{mg}$ gallic acid eq. $/ \mathrm{ml} \mathrm{ME}$ than $\mathrm{B}$ (9.08 mg gallic acid eq. /ml ME), but not significantly different. In WE versus ME, the TP concentrations of A and B were 2.4 and 3.8 times lower, respectively, similarly to the differences observed in UV spectral data.

Tab. 1

Comparative evaluation of total phenolics (expressed in gallic acid/ $\mathrm{ml} \mathrm{ME}$ ) in plant extracts (1-6) and herbal supplements A and B, using Vis spectrometry ( VIS) and FTIR, calculated from the peak intensity at $1743 \mathrm{~cm}^{-1}\left(\mathrm{I}_{1732}\right)$. The values represent the average of duplicate measurements. Details are described in Materials and methods

\begin{tabular}{|l|c|c|c|}
\hline \multicolumn{1}{|c|}{ Sample } & $\begin{array}{c}\text { Total Phenolics } \\
\text { (mg Gallic acid /ml ME) } \\
\text { VIS }\end{array}$ & $\mathrm{I}_{1732}$ & $\begin{array}{c}\text { Total Phenolics } \text { (mg } \\
\text { GAE /ml ME) } \\
\text { (FTIR) }\end{array}$ \\
\hline Dandelion & 9.30 & 0.930 & 9.39 \\
\hline Artichocke & 9.06 & 0.906 & 9.15 \\
\hline Mary Thistle & 6.66 & 0.516 & 5.31 \\
\hline St. John's Wort & 8.38 & 0.834 & 8.42 \\
\hline Celandine & 9.17 & 0.918 & 9.27 \\
\hline Wolf's claw & 7.69 & 0.764 & 7.71 \\
\hline Product A & 9.37 & 0.950 & 9.58 \\
\hline Product B & 9.08 & 0.890 & 8.98 \\
\hline
\end{tabular}

\section{FTIR fingerprints and quantification}

Fig. 2 shows the FTIR overlapped spectra $\left(650-4000 \mathrm{~cm}^{-1}\right)$ of methanol extracts corresponding to the six individual plants : the fingerprints of Dandelion Artichokes and Milk Thistle (Fig.2A), used to obtain A mix and the fingerprints of St. John's Wort, Celandine and Wolf's claw (Fig.2B), used to obtain the B mix. Fig. 2 shows also a comparative FTIR fingerprint of $\mathrm{A}$ and $\mathrm{B}$ in $\mathrm{ME}$ (Fig.2C) and WE (Fig.2D).
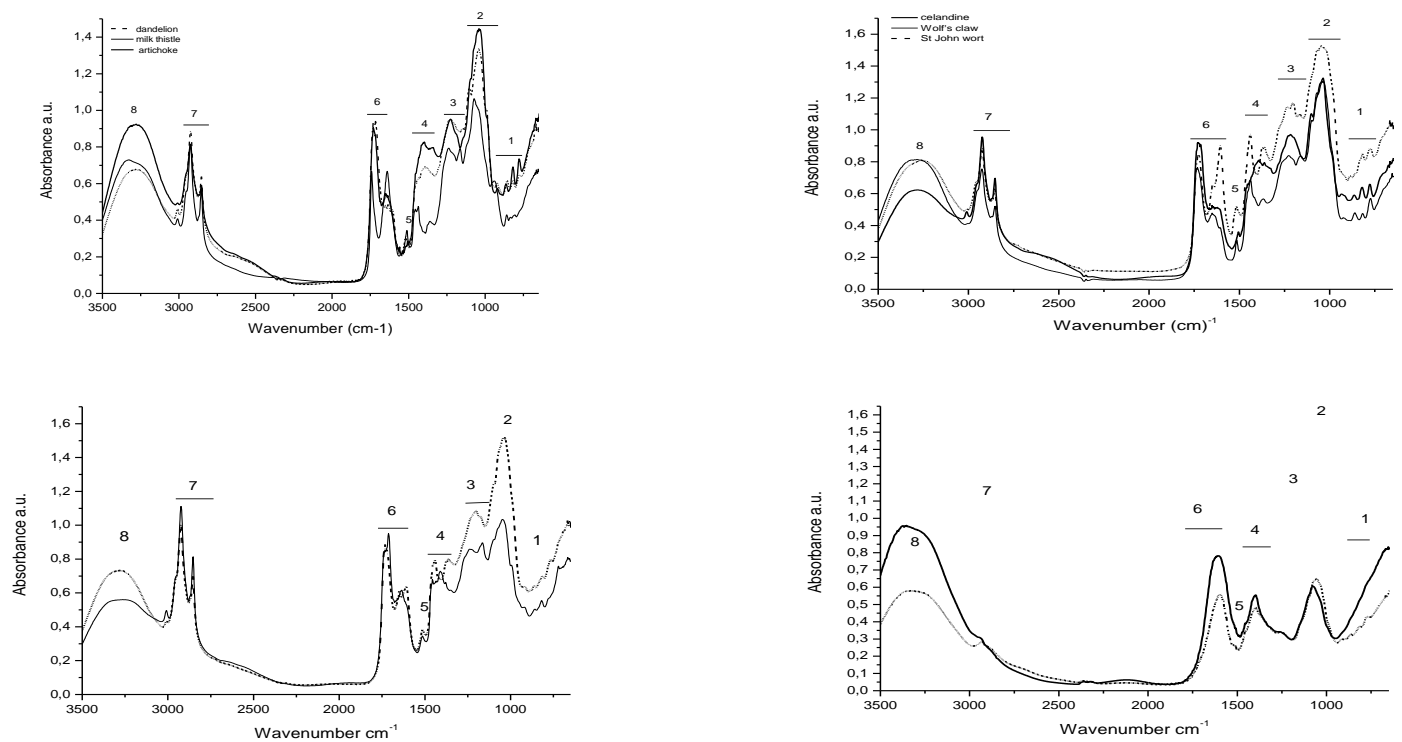

Fig. 2. FTMIR overlapped spectra $\left(650-4000 \mathrm{~cm}^{-1}\right)$ of ME of the six individual plants : upper left Dandelion, Artichocke and Milk Thistle , upper right- St. John's Wort, Celandine and Wolf's claw, comparatively with ME spectra of herbal supplements in ME ( down, left) and WE ( down, right) for A (solid line) and B (dots) 
One can identify in the methanolic extracts, eight specific regions (marked 1-8 in the figure) corresponding to functional groups which can be found in these extracts, and attributed to stretching and bending IR vibrations.

Tab. 2

Mean values of the peak absorption intensities corresponding to the 8 wavenumber areas of FTIR spectra (4000- $650 \mathrm{~cm}^{-1}$ ), for herbal supplements A and B, in water (WE) and methanolic (ME) extracts

\begin{tabular}{|c|c|c|c|c|c|}
\hline \multirow{2}{*}{\multicolumn{2}{|c|}{$\begin{array}{c}\text { Wavenumber area }\left(\mathrm{cm}^{-1}\right) \\
\text { / Samples }\end{array}$}} & \multicolumn{2}{|c|}{ WE } & \multicolumn{2}{|c|}{$\mathrm{ME}$} \\
\hline & & $\mathrm{A}$ & $\mathrm{B}$ & $\mathrm{A}$ & $\mathrm{B}$ \\
\hline \multicolumn{2}{|l|}{$3300-3350(8)$} & 0.95 & 0.58 & 0.55 & 0.74 \\
\hline \multicolumn{2}{|l|}{$2800-3000(7)$} & 0.32 & 0.29 & 1.15 & 1.01 \\
\hline \multirow[t]{2}{*}{$1600-1750(6)$} & $1732 \mathrm{~cm}^{-1}$ & 0.82 & 0.56 & 0.95 & 0.89 \\
\hline & $1620 \mathrm{~cm}^{-1}$ & 0 & 0 & 0.66 & 0.67 \\
\hline \multicolumn{2}{|l|}{$1500-1520(5)$} & 0.31 & 0.25 & 0.35 & 0.38 \\
\hline \multicolumn{2}{|l|}{$1300-1440(4)$} & 0.55 & 0.48 & 0.75 & 0.85 \\
\hline \multicolumn{2}{|l|}{$1170-1230(3)$} & 0.28 & 0.46 & 0.91 & 1.15 \\
\hline \multicolumn{2}{|l|}{$1000-1100(2)$} & 0.59 & 0.65 & 1.05 & 1.55 \\
\hline \multicolumn{2}{|l|}{$<1000 \quad$ (1) } & 0.65 & 0.62 & 0.78 & 0.85 \\
\hline
\end{tabular}

Tab. 2 presents the mean values of the peak absorption intensities corresponding to the 8 wave number areas of FTIR spectra $\left(4000-650 \mathrm{~cm}^{-1}\right)$, for herbal supplements A and B, in water (WE) and methanol (ME) extracts.

The fingerprint region was localized between 900 and $1750 \mathrm{~cm}^{-1}$ (areas 1-6). Area 1 $\left(<1000 \mathrm{~cm}^{-1}\right)$ corresponds to C-H bending vibrations found in isoprenoids, area 2 (997-1130 $\left.\mathrm{cm}^{-1}\right)$ to stretching vibrations $\mathrm{C}-\mathrm{O}$ of glucoside bonds, while area $3\left(1150-1270 \mathrm{~cm}^{-1}\right)$ corresponds to stretching vibrations of carbonyl C-O or O-H bounds. Area $4\left(1300-1450 \mathrm{~cm}^{-}\right.$ ${ }^{1}$ ) is attributed to stretching vibrations $\mathrm{C}-\mathrm{O}$ (amide) and $\mathrm{C}-\mathrm{C}$ bonds from phenyl groups, while area $5\left(1500-1520 \mathrm{~cm}^{-1}\right)$ to aromatic domain and N-H bending vibrations. Area 6 (1600-1750 $\mathrm{cm}^{-1}$ ), is a complex one corresponding to bending vibrations $\mathrm{N}-\mathrm{H}$ (amino acids), $\mathrm{C}=\mathrm{O}$ stretching (from aldehydes and ketones, esters) as well to carboxyl groups from free acids $\left(1710 \mathrm{~cm}^{-1}\right)$ or esters $\left(1740 \mathrm{~cm}^{-1}\right)$. The fingerprint of each individual plant, as well for $\mathrm{A}$ and B extracts in ME was specific and different in these 1-6 areas (Fig.1). For A and B in WE, many of the specific peaks were lost, the fingerprint was less complex and significantly different comparing with ME.

The area 7 (2800-3000 $\left.\mathrm{cm}^{-1}\right)$ corresponding to $\mathrm{C}-\mathrm{H}$ stretching vibrations specific to $\mathrm{CH}_{3}$ and $\mathrm{CH}_{2}$ from lipids, metoxy derivatives, was identified only in $\mathrm{ME}$ and not in WE, being a good indicator of the presence of lipophilic compounds in the extract. Area 8 (3000$3600 \mathrm{~cm}^{-1}$ ) corresponds to stretching vibrations of $\mathrm{OH}$ groups (from water, alcohols, phenols, carbohydrates, peroxides) as well from amides $\left(3650 \mathrm{~cm}^{-1}\right)$, and was not considered for the fingerprint analysis.

Comparing the fingerprints of the six plants, we noticed differences between the peaks 23,4 and 6. Dandelion, artichocke and St John had high intensities of area 2, which can be correlated with their higher concentration in soluble phenolics (glucosides). The ME of while milk thistle and wolfs'claw had 2 peaks in the area 6 (at around 1610 and $1732 \mathrm{~cm}^{-1}$ ) while the other plants had only one peak, at around $1732 \mathrm{~cm}^{-1}$. The difference can be correlated with the higher concentration in lipid components of milk thistle and wolfs'claw. The fingerprints of A and B supplements differ in the regions 2-4, where B shows higher intensities, correlated with stretching vibrations of $\mathrm{C}-\mathrm{O}$ bonds from glucosides and esters.

In order to validate these measurements, the FTMIR spectra of pure gallic acid, alone or mixed with dandelion extract, identifying the most specific peaks corresponding to 
phenolics, which were located at 1610 and $1732 \mathrm{~cm}^{-1}$. The peak intensities at 1610 and 1732 $\mathrm{cm}^{-1}$ were better correlated and indicated lower phenolic concentrations for plants no. 3 (milk thistle) and 6 (wolf's claw), in agreement with the spectrometry data. According to the calibration curve made with gallic acid, the best correlation IR absorption intensity $=\mathrm{f}$ ( gallic acid concentration) was found at $1732 \mathrm{~cm}^{-1}$ with a $\mathrm{R}^{2}$ value of 0.994 , then we used the curve equation $\mathrm{y}=0.0819 \mathrm{x}+0.0184$ to calculate the total phenolics based on the peak intensity at $1732 \mathrm{~cm}^{-1}$ (Zavoi, 2011b).

As shown in Tab.1, the concentrations of total phenolics (expressed in mg Gallic acid/ $\mathrm{ml} \mathrm{ME}$ ) in plant extracts (1-6) and herbal supplements A and B were compared using Vis spectrometry (VIS) and FTIR, considering the peak intensity at $1732 \mathrm{~cm}^{-1}$. A significant $(\mathrm{p}<0.001)$ correlation factor $\left(\mathrm{R}^{2}=0.979\right)$ was obtained, these findings showing that FTMIR absorption intensity at $1732 \mathrm{~cm}^{-1}$ is a reliable parameter and FTMIR can offer an adequate evaluation of the phenolics concentration in medicinal plants or herb supplements.

To summarize the data presented in this study showed that UV-Vis spectrometry achives a general fingerprint and quantification of phenolics as biomarkers of herbal formulas (A and B). The FTMIR fingerprint was very useful in discriminating the specific peaks of functional groups of molecules from individual plants and mixtures, reveals differences between methanolic and water extract, which had a different pattern and composition, in good agreement with the UV-Vis data.

Using Vis spectrometry coupled with FTIR based on peak intensities at $1732 \mathrm{~cm}^{-1}$ and calibrated with gallic acid, the total phenolics ranged from 5.31 to $9.58 \mathrm{mg}$ gallic acid eq/ml ME, with a positive and significant correlation between the two methods $\left(R^{2}=0.979\right)$. The TP concentrations were 2.5 to 4 times lower in WE comparative to ME of products $\mathrm{A}$ and B.

This study demonstrated that herbal supplements, based on medicinal plant ingredients can be adequately characterized for their quality and safety by combined UV-Vis spectrometry and FTIR spectrometry. These easy-to-handle, cheaper and fast methods gives valuable information about the main biomarkers of authenticity, their concentration, the minor components, the key-molecules of authenticity and to identify the adulteration of herbal supplements.

Our studies can contribute to data cumulated in the European databank of Natural resources, including medicinal plant composition and many derived formulas, many of them known as "traditional medicines". These techniques can offer a good and efficient tool to demonstrate the quality and authenticity of herbal supplements and predict their potential for biomedical applications.

Acknowledgement. This study acknowledges the partial financial support for CCDBIODIATECH from POSCCE project O 2.3.2. nr. 1033/27632 ( 2011-2012).

\section{REFERENCES}

1. Baranska, M. and H. Schultz (2006). Application of Infrared and Raman spectroscopy for analysis of selected medicinal and spice plants. J. Med. Spice Plant 2:72-80

2. Barnes, J. et al. (2001). St. John's wort (Hypericum perforatum L): a review of its chemistry, pharmacology and clinical properties. J. Pharmacol. 53: 583-600

3. Ding, T. et al. (2001). Determination of active component in silymarin by RP-LC and LC/MS. J. Pharm. Biomed. Anal. 26:156-201

4. Duron, R.R. et al. (2009). Development and Validation of Thin-Layer Chromatographic Methods for Quality Control of Herbal Products. Acta Chromatographica 21: 203-215 
5. Fan, X.H. et al. (2006). Multiple chromatographic fingerprinting and its application to the quality control of herbal medicines. Anal. Chim. Acta. 555:217-224

6. Giri, L. et al. (2010). Chromatographic and spectral fingerprinting standardization of traditional medicines: an overview as modern tools. Res. J. Phytochem. 4:234-241

7. Gong, F. et al. (2006). Variable selection for discriminating herbal medicines with chromatographic fingerprints. Anal. Chim. Acta 572:265-271

8. Gong, F. et al., (2009). Quality Assessment of Herbal Medicine with Chromatographic Fingerprint, J. Drug Addition, Education and Eradication 4: 257-302

9. Liang, Y.Z. et al. (2004). Review: Quality control of herbal medicines. J. Chromatography B 812: 53-70

10.Mattoli, L. et al. (2011). A metabolite fingerprinting for the characterization of commercial botanical dietary supplements. Metabolomics 7:437-445

11.Minakhmetov, R.A. et al., (2001). Analysis of Flavonoids in Silybum marianum Fruit by HPLC. Chem. Nat.Comp. 37:318-321

12.Mulinacci, N. et al. (1999). HPLC-DAD and TLC-Densitometry for quantification of hypericin in Hypericum perforatum L. Extracts. Chromatographia 49: 197-201

13.Pedersen J.A. and B. Øllgaard (1982). Phenolic acids in the genus Lycopodium. Biochem. Systematics and Ecology 10:3-9

14.Ramawat, K.G. et al., (2009). In: Ramawat KG (ed.) Herbal Drugs: Ethnomedicine to Modern Medicine p. 7-32. Springer-Verlag, Berlin, Heidelberg

15.Santos-Buelga, C. and Williamson G. (Eds.) (2003) Methods in polyphenol analysis.The Royal Society of Chemistry, Cambridge

16.Scalbert, A. et al., (2005). Dietary polyphenols and the prevention of diseases. Crit. Rev. Food. Sci. Nutr. 45: 287-306

17.Schütz K. et al., (2005). Characterization of phenolic acids and flavonoids in dandelion (Taraxacum officinale) root and herb by high-performance liquid chromatography/electrospray ionization mass spectrometry. Rapid Commun. Mass Spectrom.19:179-186

18.Singleton, V.L. (1999). Analysis of total phenols and other oxidation substrates and antioxidants by means of Folin-Ciocalteu reagent. Methods Enzymol. 299:152-178

19.Socaciu, C. et al., (2009). Complementary advanced techniques applied for plant and food Authentication. Czech J Food Sci 27:S70-S75

20.Spiridon, I. et al., (2011). Total phenolic content and antioxidant activity of plants used in traditional Romanian herbal medicin. Central Eur J Biol 6: 388-396

21.Zavoi, S. (2011a). PhD dissertation, USAMV Cluj-Napoca

22.Zavoi, S. et al. (2011b). Comparative fingerprint and extraction yield of medicinal herb phenolics with hepatoprotective potential, as determined by UV-Vis and FT-MIR spectroscopy, Not Bot Hortiagrobot Cluj-Napoca, 39(2): 82-89

23. Verpoorte, R. (2009). Medicinal Plants: A Renewable Resource for Novel Leads and Drugs, in Herbal Drugs: Ethnomedicine to Modern Medicine, (Ramawat K.G., ed.) p. 1-5, Springer-Verlag, Berlin Heidelberg

24.Wang, M.F. et al., (2003). Analysis of antioxidative phenolic compounds in artichoke Cynara scolymus L. J Agr Food Chem 51:601-608

25.Wichtl, M. (2004). Herbal drugs and phytopharmaceuticals. $3^{\text {rd }}$ ed. Boca Raton: Medpharm p. $152-156$

26. Yadav, N.P. and V.K. Dixit (2008). Recent approaches in herbal drug standardization. Int. J. Integr. Biol. 2:195-203 